\title{
Elective procedures for prostate cancer in the time of Covid-19: a multidisciplinary team experience
}

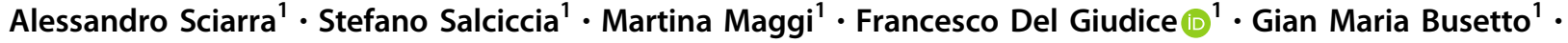 \\ Daniela Musio ${ }^{2} \cdot$ Antonio Ciardi $^{3} \cdot$ Carlo Catalano $^{3} \cdot$ Enrico Cortesi $^{3} \cdot$ Valeria Panebianco $\mathbb{C}^{3}$
}

Received: 3 April 2020 / Revised: 19 April 2020 / Accepted: 6 May 2020 / Published online: 18 May 2020

(c) Springer Nature Limited 2020

\begin{abstract}
On March 29th 2020, 97,689 cases of COVID-19 have been diagnosed only in Italy, with 73,880 actually positive cases, a daily increase of 3815 cases, 27,386 hospitalized and 3906 patients in intensive care units, causing a total of 10,779 known deaths. In all urological departments, quickly inpatient and outpatient services have been significantly reduced. Even in this COVID-19 situation, urological neoplasm care must go on, but significant changes need to be made in the way some care is delivered. We compared diagnostic and therapeutic elective procedures requested and performed for PC management from our multidisciplinary team (MDT) during 1 month activity in the highest national level of COVID-19 infection (March 2020) and under restrictions for all the population, with the management performed in a no-COVID-19 month (March 2019) 1 year ago. The only management that did not received a significant reduction are medical therapies for advanced hormone sensitive (HS) or castration resistant (CR) PC. We describe our MDT identifications of elective undeferrable PC management in this COVID-19 time. These suggestions have been considered for a country (ITALY) under a rapid increase of COVID-19 cases and complications, but in a region with an actual lower impact (2914 actual positive and 1079 hospitalized cases) from the infection and in an hospital not completely converted to COVID-19 management. Indications should be different and restricted only to emergencies on the basis of COVID-19 pandemic situation and hospital involvement.
\end{abstract}

The pandemic acute respiratory syndrome coronavirus 2 (SARS-CoV-2) named COVID-19 [1] is generating a severe health emergency in all the world but in particular in some countries. On March 29th 2020, 97,689 cases of COVID-19 have been diagnosed only in Italy, with 73,880 actually positive cases, a daily increase of 3815 cases, 27,386 hospitalized and 3906 patients in intensive care units, causing a total of 10,779 known deaths.

Supplementary information The online version of this article (https:// doi.org/10.1038/s41391-020-0240-4) contains supplementary material, which is available to authorized users.

Stefano Salciccia

stefano.salciccia@uniroma1.it

1 Department of Urology, University Sapienza, Rome, Italy

2 Department of Radiological Sciences, Oncology \& Pathology, University Sapienza, Rome, Italy

3 Department of Radiology, Oncology and Pathology, Sapienza University of Rome, Rome, Italy
In all urological departments, quickly inpatient and outpatient services have been significantly reduced in preparation for a possible surge of COVID-19-related admissions and also to reduce the possibility of contacts and disease transmission.

Even in this COVID-19 situation, urological neoplasm care must go on, but significant changes need to be made in the way some care is delivered $[2,3]$. Our clinical practice, through all diagnostic and therapeutic procedures routinely used for our patients, can negatively affect dispositions organized to contain COVID-19 disease [4-6].

Prostate cancer (PC) is a heterogeneous neoplasm including no-clinically significant or slowly progressive diseases but also high risk, clinically significant and life influencing cancers. All guidelines specified that the best management of PC is provided by a multidisciplinary team (MDT) organization able to decide the best diagnostic and therapeutic routes for our patients. This is particularly true in this time of COVID-19, where choices for PC are not only influenced by oncologic criteria.

PC teams should continue to have online consultations among specialists, so to do the best they can to deliver 
care to those most in need. Our challenge is to find a balance between undertreating people with cancer, resulting in more clinically significant progression and future deaths from the disease in the medium or long time and increasing COVID-19 transmission, complications and death in a short time. In this evaluation we must consider PC characteristics but also the vulnerable patient population affected by this neoplasm $[4,5]$.

Since 2012 our MDT gives a multidisciplinary management to a population at risk or with a diagnosis of PC. In this COVID-19 time we are continuing to work in an Italian region with relatively lower number of COVID-19 cases when compared with the North of Italy and in a department and hospital that is not completely converted in COVID-19 units but that could be most involved in case of a surge.

In Supplementary Fig. 1, we compared diagnostic and therapeutic elective procedures requested and performed for PC management during 1 month activity at the time of the highest national level of COVID-19 infection (March 2020) and under restrictions for all the population, with the management performed in a no-COVID-19 month (March 2019) 1 year ago.

As expected we describes an almost complete interruption of PSA determination in particular for an early diagnosis but also in the follow-up after PC treatments. Outpatient consultations were almost completely converted in online or phone interviews.

Similarly prostate biopsy procedures were significantly (Student's $t$ test) limited to suspected high volume or advanced stage diseases. In particular, we prefer to obtain a multiparametric magnetic resonance (mMR) in high risk cases so to better select them for an undeferrable biopsy [7].

The rate of primary treatments for PC significantly reduced during this COVID-19 month, either for radical prostatectomy (RP) procedures (63.6\% reduction) or for radiotherapy (RT) (84.6\% reduction). The reduction in RP procedures is also due to a limited availability of anesthesiologists and ventilators in this time whereas the reduction in radiotherapy is also justified by the possibility to prolong neoadjuvant hormone therapies. In the consideration to shift surgical treatment to RT we should consider the need to repeat access to the hospital for RT delivery and the combination with hormone systemic therapies [4].

The only management that did not received a significant reduction is systemic therapy for advanced hormone sensitive or castration resistant (CR) PC. In the choice for an initial treatment strategy for CRPC, considering the absence of a demonstrated advantage of one therapeutic sequence on the others, androgen receptor-targeted agents could be preferred to chemotherapy considering the possibility to avoid hospital administration.

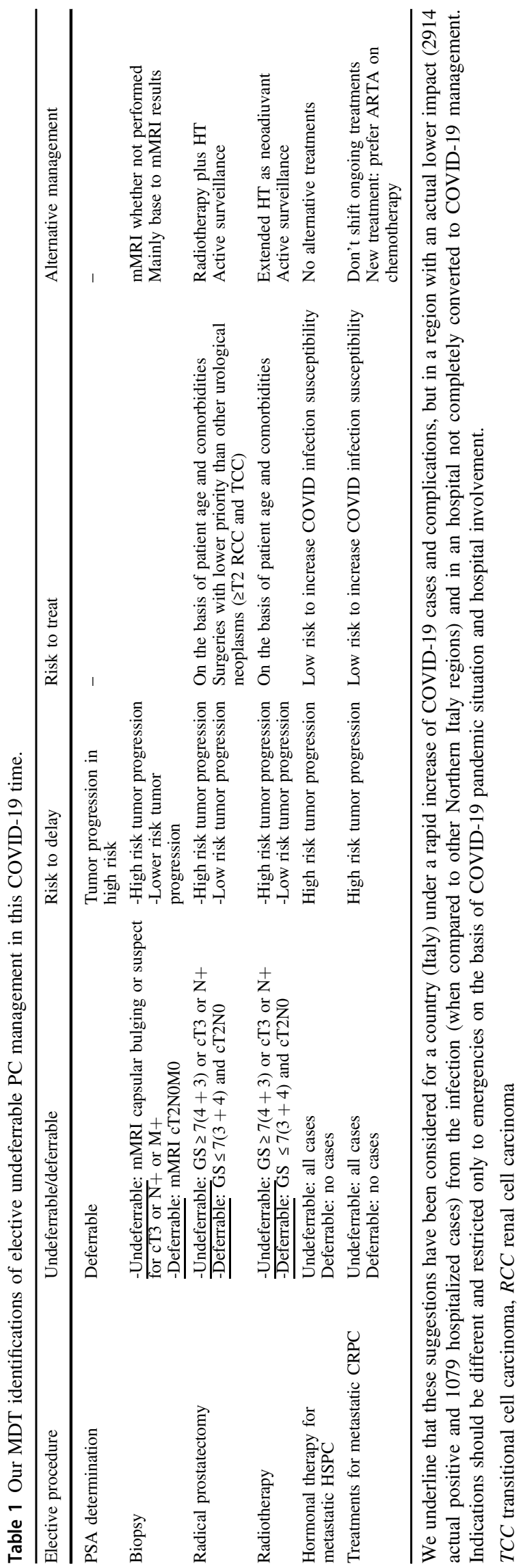


Unavoidably, COVID-19 pandemic will delay PC prevention strategies and all elective treatments and controls. Hospitals directly involved in COVID-19 treatment completely suspended all activities related to PC management. Several hospitals not directly involved in COVID-19 treatment equally reduced all urological activities only to emergencies. Compared to other urological neoplasms, PC seems to be less undeferrable in the management. However, even in this time, PC care for clinically significant and high risk cases should go on, correctly selecting cases and procedures. Hoping that COVID-19 pandemic will remain a short time situation, the way we treat PC over the next months will change and will influence medium time results.

It is possible that [8] cancer patients have a significantly higher risk of complications resulting in intensive care admission or death when infected by COVID-19, particularly if they recently performed chemotherapies or surgery. Also for this reason we should avoid or delay those treatments that demonstrated small benefits in terms of clinical progression or cancer related survival.

Table 1 describes our MDT identifications of elective undeferrable PC management in this COVID-19 time. PC in several cases is a chronic and slowly progressive disease, alternative treatment options are often available, the population involved is generally particularly vulnerable to this infection for aging and co-morbidities. Early diagnosis of PC can absolutely be delayed in this emergency situation; primary treatments should be carefully evaluated considering the risk for a short term progression and the possible alternatives (active surveillance, surgery, or radiotherapy) [9]. Systemic treatments for high risk and advanced stages should not be stopped as the balance of potential harms that may result from delaying treatment versus the potential not demonstrated benefits of preventing COVID-19 infection and complications could be negative. In this pandemic situation a MDT evaluation for the management of our patients should be implemented where possible in order to assign the most appropriate priority to patients, better balancing oncologic and COVID-19 restraint needs.

\section{Compliance with ethical standards}

Conflict of interest The authors declare that they have no conflict of interest.

Publisher's note Springer Nature remains neutral with regard to jurisdictional claims in published maps and institutional affiliations.

\section{References}

1. Livingston E, Bucher K. Coronavirus Disease 2019 (COVID-19) in Italy. JAMA. 2020. https://doi.org/10.1001/jama.2020.4344.

2. Kashi AH. COVID-19, urologists and hospitals. Urol J. 2020. https://doi.org/10.22037/uj.v0i0.6064.

3. Nowroozi A, Amini E. Urology practice in the time of COVID-19. Urol J. 2020. https://doi.org/10.22037/uj.v0i0.6065.

4. Ficarra V, Novara G, Abrate A, Bartoletti R, Crestani A, De Nunzio $\mathrm{C}$, et al. Urology practice during COVID-19 pandemic. Members of the Research Urology Network (RUN). Minerva Urol Nefrol. 2020. https://doi.org/10.23736/S0393-2249.20.03846-1.

5. Chan MC, Yeo SEK, Chong YL, Lee YM. Stepping forwards: urologists' efforts during the COVID-19 outbreak in Singapore Eur Urol. 2020 Mar. pii: S0302-2838(20)30145-7. https://doi.org/10. 1016/j.eururo.2020.03.004.

6. Stensland KD, Morgan TM, Moinzadeh A, Lee CT, Briganti A, Catto J, et al. Considerations in the triage of urologic surgeries during the COVID-19 pandemic. Eur Urol. 2020. [Epub ahead of print]

7. Maggi M, Panebianco V, Mosca A, Salciccia S, Gentilucci A, Di Pierro G, et al. Prostate imaging reporting and data system 3 category cases at multiparametric magnetic resonance for prostate cancer: a systematic review and meta-analysis. Eur Urol Focus. 2019. pii: S2405-4569(19)30169-5. https://doi.org/10.1016/j.euf. 2019.06.014.

8. Sciarra A, Gentilucci A, Salciccia S, Von Heland M, Ricciuti GP, Marzio V, et al. Psychological and functional effect of different primary treatments for prostate cancer: a comparative prospective analysis. Urol Oncol. 2018;36:340.e7-340.e21.

9. Xia Y, Jin R, Zhao J, Li W, Shen H Risk of COVID-19 for cancer patients. Lancet Oncol. 2020. pii: S1470-2045(20)30150-9. https:// doi.org/10.1016/S1470-2045(20)30150-9. 